\title{
L'analyse du discours philosophique : bilan et perspectives
}

Philosophical Discourse Analysis: Results and New Perspectives

Frédéric Cossutta et Dominique Maingueneau

\section{(2) OpenEdition}

Journals

Édition électronique

URL : https://journals.openedition.org/aad/2981

DOI : 10.4000/aad.2981

ISSN : $1565-8961$

Éditeur

Université de Tel-Aviv

Référence électronique

Frédéric Cossutta et Dominique Maingueneau, «L'analyse du discours philosophique : bilan et perspectives », Argumentation et Analyse du Discours [En ligne], 22 | 2019, mis en ligne le 15 avril 2019, consulté le 07 décembre 2022. URL : http://journals.openedition.org/aad/2981 ; DOI : https://doi.org/ 10.4000/aad.2981

Ce document a été généré automatiquement le 29 septembre 2020.

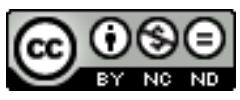

Creative Commons - Attribution - Pas d'Utilisation Commerciale - Pas de Modification 4.0 International - CC BY-NC-ND 4.0

https://creativecommons.org/licenses/by-nc-nd/4.0/ 


\title{
L'analyse du discours philosophique : bilan et perspectives
}

\author{
Philosophical Discourse Analysis: Results and New Perspectives
}

Frédéric Cossutta et Dominique Maingueneau

\section{Analyse du discours et analyse du discours philosophique}

1 En 1995 est paru un numéro de la revue Langages intitulé «l'Analyse du discours philosophique ", dirigé par F. Cossutta. C'est, à notre connaissance, la première publication consacrée à ce sujet qui se réclame de l'analyse du discours ${ }^{1}$. Le présent numéro d'Argumentation et Analyse du discours reprend le flambeau, presque un quart de siècle plus tard, en s'appuyant comme le précédent sur les travaux du Groupe de recherche sur l'analyse du discours philosophique (GradPhi), fondé au Collège International de Philosophie en 1994 et qui n'a cessé ses activités depuis².

2 Le terme d'" analyse du discours philosophique » est quelque peu ambigu, mais d'une ambiguïté féconde, cette expression ayant deux acceptions : elle peut prendre le sens général d'une investigation par analyse des textes philosophiques qui entend rompre avec l'histoire de la philosophie ou l'étude monographique des doctrines, sans qu'on puisse préjuger de la nature de l'investigation proposée. Ainsi certains membres du GradPhi se reconnaissent tout à fait dans une "Analyse (du discours philosophique) ", sans nécessairement se référer à ce qui serait la seconde acception de cette expression ${ }^{3}$. En revanche, si l'on accentue "Analyse du discours» dans l'expression "Analyse du discours (philosophique)", on se réfère à un champ disciplinaire spécifique et historiquement situé dans le paysage des sciences humaines et sociales ${ }^{4}$. Dans cette seconde acception, une " Analyse du discours philosophique » fait de la philosophie un type de discours parmi d'autres, susceptible d'être exploré grâce aux méthodes et aux 
catégories issues de l'Analyse du discours, le terme de discours étant nettement distingué de celui de texte ${ }^{5}$. Nous avons délibérément choisi de privilégier cette acception disciplinaire en cohérence avec l'esprit de la revue, quitte à la soumettre à certaines analyses critiques.

En abordant la philosophie comme "discours", on espère renouveler la compréhension de l'activité philosophique, mais aussi enrichir les catégories et les méthodes de l'analyse du discours.

\subsection{Comment expliquer les résistances suscitées par une approche de la philosophie comme discours?}

4 L'analyse du discours a vocation à aborder les manifestations du discours dans toute leur diversité. Pourtant, aujourd'hui encore aborder la philosophie à travers des problématiques d'analyse du discours continue à susciter des réticences, tant du côté des philosophes que du côté des analystes du discours.

Dans leur immense majorité, ces derniers ne s'intéressent pas au discours philosophique. En France, le succès dans les années 1960-1970 des spéculations sur « l'écriture » ou le «Texte » majuscule a radicalisé le présupposé selon lequel, en droit, les textes littéraires ou philosophiques doivent faire l'objet d'approches incommensurables avec celles des textes « ordinaires ». Les analystes du discours ont surtout étudié les corpus délaissés par les facultés de lettres. Une tendance renforcée par la mondialisation des perspectives micro-sociologiques et interactionnistes, naturellement tournées vers l'oralité conversationnelle; ce qui est difficilement compatible avec l'étude de corpus philosophiques. Intégrer ces derniers dans le champ de l'analyse du discours ne peut donc qu'avoir un coût élevé pour ces chercheurs, qui seraient obligés de repenser un certain nombre de leurs présupposés théoriques et méthodologiques.

6 La seconde raison qui peut expliquer la réticence des analystes du discours tient aux ambigüités des relations entre analyse du discours et philosophie. Comme le montrent ici les contributions de $\mathrm{C}$. Thevenet et $\mathrm{M}$. Vallespir, le partage entre le discours objet (la philosophie) et le discours qui appréhende cet objet (l'analyse du discours) n'est pas aussi net qu'on pourrait le penser, au risque de brouiller les identités disciplinaires. D'ailleurs, l'Ecole française d'analyse du discours est largement tributaire, dans ses origines et ses fondements, de certains présupposés philosophiques, comme le montre le recours au terme althussérien de "formation discursive ", aux critiques par Foucault des catégories de sujet et d'auteur ou inversement à une théorie du sujet lorsqu'on se réfère à l'énonciation selon Benveniste. Il peut donc sembler compliqué de mobiliser l'analyse du discours pour étudier un objet qui, par ailleurs, est en droit d'en interroger les présupposés épistémologiques.

7 On peut expliquer de façon symétrique les raisons pour lesquelles les philosophes, de leur côté, se méfient, pour ne pas dire ignorent ce nouveau type d'approche de leur discipline ${ }^{6}$. On peut d'autant plus s'en étonner qu'ils s'intéressent depuis les origines à la question du langage et qu'ils s'interrogent, pour des raisons nécessaires, sur leur propre discours, parlant tantôt de «langage de la philosophie ", tantôt de «style des philosophes ", selon qu'ils considèrent la philosophie comme une langue de spécialité ou comme un ensemble d'œuvres singulières. 
Mais la notion même de "discours philosophique », qui place la philosophie au milieu d'autres types de discours, et l'appartenance de l'analyse du discours aux sciences humaines et sociales éveillent la méfiance des philosophes, qui ramènent les approches en termes de discours à des approches, pour eux réductionnistes, de type sociologique

La philosophie, en effet, même si elle a renoncé en grande partie à l'hégémonie qu'une longue tradition lui a conférée, prétend, dans la mesure où elle explicite les conditions de véracité de son propre discours, pouvoir soumettre les autres formes de discours à son examen critique. De même, elle dénie toute légitimité aux disciplines qui voudraient la prendre pour objet, acceptant tout au plus les apports de disciplines dont le statut ancillaire ne touche pas à l'essence même de la philosophie.

\subsection{La diversité des formes d'« analyse » de la philosophie}

L'analyse du discours philosophique se distingue des approches sociologiques comme de l'histoire des doctrines, mais elle n'est pas seule à avoir été sensible aux dimensions langagières ou institutionnelles de la philosophie. Elle n'occupe qu'une portion de champ au sein des analyses du discours, et d'autres disciplines relevant de la philosophie elle-même, de la sociologie ou des sciences du langage (stylistique, sémiotique, linguistique textuelle, etc.), sont à même de prendre en charge la textualité philosophique.

\subsubsection{Approches philosophiques : herméneutique, déconstruction, philosophie analytique}

11 Nombre de travaux conduits par des historiens de la philosophie ou des philosophes sensibles à la question des structures et des formes d'expression ont déplacé la lecture que l'on peut faire des grands textes. Que l'on songe à E. Souriau (1939), M. Gueroult (1976) ' , V. Goldschmidt (1971), J. Vuillemin (1984), G.-G. Granger (1988), A. Robinet (1996), M. Serres (1968). Les travaux de P. Hadot, en philosophie antique, restituent, sous les textes déposés dans leur monumentalité posthume, les " exercices spirituels » qui leur ont donné vie, ce qui entre en résonance avec une théorie des actes de langage (Hadot 1987). On peut penser aussi à des approches plus spécifiques sur l'écriture des philosophes (Loraux 1993, Soulez 2003, Botet 2006), sur l'analyse des systèmes ${ }^{8}$, ou sur le rapport entre littérature et philosophie (Macherey 1990, Sabot 2001, Clément 2000, $2005)^{9}$.

Par ailleurs, certaines philosophies prennent directement en charge la dimension textuelle ou la logique des énoncés philosophiques, comme c'est le cas en particulier avec l'herméneutique, marquée par les travaux de Gadamer sur les Dialogues de Platon (Gadamer 1976) ou de Ricœur qui définit ce qu'est un texte dans Du texte à l'action (1986) mais ne se pose pas explicitement la question du statut du texte philosophique en tant que tel. La perspective est très différente (en apparence du moins ${ }^{10}$ ), dans la déconstruction opérée par Derrida à même les textes, dont il offre une méthode de lecture très puissante, intriquée dans un propos qui reste de part en part philosophique, dans sa prétention même à subvertir le philosophique (Derrida 1998, Maingueneau et Vallespir (éds) 2015).

Dans le domaine anglo-saxon, à côté des instruments logiques utilisés par les néopositivistes comme Carnap, la philosophie analytique, telle qu'elle est illustrée par 
G. Ryle sur Descartes par exemple, propose des méthodes d'analyse qui, partant de la norme du langage ordinaire, détectent les « erreurs de catégories » et les " expressions systématiquement trompeuses » dans les énoncés philosophiques (Ryle 1971, 1978).

14 Mais les trois courants que nous venons de mentionner (herméneutique, déconstruction, philosophie de l'analyse) cherchent moins à expliquer comment "fonctionne » le discours philosophique qu'à en comprendre les dysfonctionnements ou à en parasiter l'inconsistance par le redoublement du commentaire. Ce qui les conduit à très vite substituer à l'analyse critique un programme de reconstruction d'une philosophie nouvelle, qui peut aller jusqu'à associer dans le même pli l'analysant et l'analysé, comme le fait la déconstruction derridienne.

\subsubsection{Sociologie de la philosophie ou analyse du discours?}

15 Les études sociologiques sur les carrières ou les appareils universitaires ne font pas l'objet du même rejet que l'analyse du discours ${ }^{11}$, car elles sont reléguées à l'extérieur de ce qui est considéré comme l'essentiel par les philosophes : les doctrines. L'analyse du discours se distingue d'une sociologie de la philosophie, cette dernière fût-elle, comme c'est le cas dans les travaux de J.-L. Fabiani, soucieuse de sortir d'un raisonnement en termes de «facteurs sociaux » qui conditionneraient une philosophie dont on affirme « alternativement qu'elle est par elle-même et de part en part sociale, comme n'importe quelle autre activité humaine, ou que les éléments sociaux qu'elle peut contenir accidentellement ne jouent aucun rôle dans le cours de son développement logique » (Fabiani $2010: 20)$. Mais cette sociologie reste partiellement prise dans la dissociation entre texte et contexte, alors même qu'elle la conteste : les doctrines dans leur singularité et leurs modes d'expression passent pour une très large part à travers les mailles de ses filets ${ }^{12}$. L'analyse du discours, en revanche, précisément parce qu'elle est fortement enracinée dans les sciences du langage, peut se focaliser sur la zone que les sciences humaines et sociales ont tendance à délaisser : le mouvement par lequel texte et contexte passent l'un dans l'autre.

\subsubsection{Analyse textuelle ou analyse du discours?}

16 Certaines investigations, conduites dans le cadre des sciences du langage ont donné lieu à des développements conséquents, d'une portée heuristique certaine.

17 La théorie des opérations énonciatives d'A. Culioli a pu inspirer des transpositions de la phrase au discours et notamment au discours philosophique, comme en témoignent les travaux de M. Ali Bouacha $(1995,2000)$ ou certains textes de D. Ducard (2019).

Les perspectives stylistiques, ont permis d'étudier certaines configurations terminologiques ou figures de style comme le montre le titre significatif: Un autre Descartes. Le philosophe et son langage de A. Cahné (1980) ou le collectif Le style des philosophes (B. Curatolo J. Poirier éds 2007).

19 Après les travaux de C. Perelman et L. Olbrechts-Tyteca (2008 [1970]), R. Amossy (2000), J.-B. Grize (Grize (éd.) 1984), ou C. Plantin (2016) ont proposé de nouvelles grilles d'analyse des phénomènes argumentatifs ou persuasifs, mieux en prise sur les contraintes de la langue . J.-F. Bordron, dans le sillage de Greimas, a développé une analyse sémiotique qui prend en considération aussi bien le discours philosophique en général (Bordron 2016) que des études d'œuvres canoniques comme les Méditations métaphysiques (Bordron 1987). L'un des intérêts de sa démarche est d'associer une 
dimension iconique aux schèmes spéculatifs qui structurent les doctrines et les œuvres des philosophes (Bordron 2011).

Les différends épistémologiques qui séparent ces courants portent sur l'importance accordée à la textualité et à l'écriture dans une œuvre de pensée. Cette question a été traitée dans les travaux de linguistique textuelle de J.-M. Adam, un des enjeux étant de savoir s'il y a autonomie ou complémentarité entre les deux types d'approche. J.-M. Adam, tout en libérant un espace propre pour l'approche textuelle, en se proposant d'expliciter la diversité des formes de textualisation, est conduit à plaider pour un modèle qui associe les deux dimensions (Adam 2008, 2011). Ce courant s'est cependant rarement donné pour objet le discours philosophique ${ }^{13}$.

Le débat concernant l'importance respective à accorder au texte et au discours est aussi crucial que celui qui porte sur le rapport entre texte et contexte. L'article d'A. Lhomme dans ce numéro entend montrer que si ces deux approches sont éventuellement complémentaires, il faut respecter leur autonomie respective, la dimension textuelle n'étant pas, à ses yeux, soluble dans l'analyse du discours, alors que J.-M. Adam plaide pour leur articulation étroite et que $\mathrm{D}$. Maingueneau et $\mathrm{F}$. Cossutta pensent intégrer la dimension textuelle comme une composante à part entière de la discursivité. C'est l'objet d'un débat fécond.

L'analyse du discours philosophique, en mettant en avant la dimension énonciative, entend se situer entre ce qu'elle considère comme deux écueils : celui d'une approche purement textuelle qui, appréhendant la philosophie en immanence, tend à négliger le fait que les textes sont eux-mêmes pris dans une relation en boucle par rapport aux pratiques et aux institutions qui font vivre la philosophie. Pour autant, elle n'accepte pas non plus de réduire la part textuelle à la portion congrue au profit des stratégies d'acteurs sociaux qui produiraient des textes pour s'assurer une position de pouvoir; elle accepte encore moins toute approche qui ferait du texte philosophique un simple effet ou reflet de jeux de forces sociales.

\section{Les conditions d'une analyse du discours philosophique}

\subsection{L'énonciation philosophique comme médiation entre texte et contexte}

Le principe même d'une démarche d'analyse du discours implique de porter l'attention sur l'énonciation, qui ne se laisse enfermer ni dans une problématique du texte ni dans une problématique strictement sociologique, cette dernière fût-elle centrée sur la dimension institutionnelle et le point de vue de l'acteur. De manière constitutive, la problématique de l'énonciation n'est pas compatible avec une conception élémentaire de la relation entre «texte » et " contexte». L'instance qui soutient et se soutient de l'énonciation n'est en effet ni dans l'énoncé, ni quelque entité située hors du langage : " entre » le texte et le contexte il y a l'énonciation, « entre » l'espace de production et l'espace textuel il y a la scène d'énonciation, un « entre » qui déjoue toute extériorité immédiate.

On peut parler d' "institution discursive " (Maingueneau 2015) en s'éloignant de l'usage courant qui voit dans l'institution philosophique un certain nombre de 
phénomènes extérieurs aux doctrines: carrières, publications, enseignement, colloques, prix... Dans l'institution discursive se mêlent inextricablement l'institution comme action d'établir, processus de construction légitime, et l'institution au sens usuel d'appareils qui organisent des pratiques. Ce qui importe ici, c'est la réversibilité entre l'activité énonciative et les structures qui en sont à la fois la condition et le produit. L'activité énonciative doit gérer sa présence à la fois dans le monde qu'elle construit et dans le monde où elle se construit, qui se déploie comme dispositif de légitimation de la situation d'énonciation dont elle prétend surgir. Dès lors, on ne cherche pas, comme dans la démarche structuraliste, une théorie de "l'articulation " entre le texte et une réalité extraverbale muette : cela reviendrait à présupposer le partage même qu'on cherche à surmonter.

Il s'agit ainsi de jouer sur la double valeur d' « institution » : processus d'instauration ${ }^{14}$ légitimante et ensemble d'appareils et de règles établis. À travers le mouvement d'institution discursive s'articulent les institutions qui donnent sens à l'énonciation singulière (la structure du champ, le statut de la philosophe, les genres de texte...) et le mouvement par lequel s'institue le discours, à la fois en instaurant progressivement un certain monde dans son énoncé et en légitimant la scène d'énonciation et le positionnement dans le champ qui rend cet énoncé possible. Chaque geste créateur mobilise, qu'il le veuille ou non, l'espace qui le rend possible, et ce même espace ne tient que par les gestes créateurs qu'il rend possibles. L'œuvre, à travers le monde qu'elle configure en déployant son texte, réfléchit en les légitimant les conditions de sa propre activité énonciative. De là le rôle crucial que doit jouer la «scène d'énonciation ", qui n'est réductible ni au texte ni à une situation de communication qu'on pourrait décrire de l'extérieur. L'œuvre présuppose une scène de parole déterminée qu'il lui faut valider à travers son énoncé même. L'institution discursive est ainsi le mouvement par lequel passent l'un dans l'autre, pour s'étayer, l'œuvre et ses conditions d'énonciation.

\subsection{Diversité des approches au sein de l'analyse du discours philosophique}

Pour autant, bien que son spectre soit faible, parce qu'elle n'a pas véritablement fait école, l'analyse du discours philosophique n'est pas monolithique ${ }^{15}$. On peut rendre compte de la diversité de ses ancrages théoriques en considérant le statut que les différentes approches donnent à l'objet étudié, la philosophie.

- Tantôt l'analyste du discours s'efforce de modéliser le fonctionnement d'une zone déterminée de la production sémiotique et de l'inscrire dans l'ensemble des pratiques discursives d'une société, dans un cadre théorique où le discours philosophique n'est qu'un discours parmi d'autres. Le discours philosophique est appréhendé comme réseau de genres de discours très divers qui vont des traités sur l'ontologie aux reportages dans les magazines en passant par les commentaires, les cours de philosophie dans les lycées, les colloques, etc. L'analyse du discours n'est alors pas censée étudier des cuvres , mais des corpus ; les analystes rassemblent les matériaux qu'ils jugent nécessaires pour répondre à tel ou tel questionnement explicite, en fonction des contraintes qu'imposent les méthodes auxquelles ils recourent. La différence entre œuvre et corpus marque une frontière entre les pratiques de commentaire qui s'efforcent d'interpréter un Thésaurus de textes légués par la tradition et des approches qui relèvent des 
sciences humaines et sociales. De cette inspiration relèvent les travaux de J. Angermuller (2013 a, b), F. Cicurel, (2014), P. Delormas (2013), M. Temmar (2013); D. Maingueneau (2015) inscrit ses investigations sur le discours philosophique dans un ensemble beaucoup plus vaste, celui des « discours constituants ».

- Tantôt l'analyste du discours participe aussi de la philosophie, à condition de poser un cadre théorique capable d'expliciter et de penser rigoureusement leurs rapports ${ }^{16}$. En effet, rien n'interdit à l'analyse du discours philosophique de penser le statut de ce qui, au-delà du corpus, fait qu'une philosophie peut se donner à travers des "œuvres ", voire des «chefs d'œuvre ». Mais cela doit se faire dans les catégories de l'analyse et non à partir de quelque norme axiologique ou esthétique qui s'attacherait à évaluer $a$ priori la valeur philosophique d'une philosophie. Pour F. Cossutta par exemple, la visée de vérité exclusive, à l'origine de la concurrence entre les philosophies, doit être provisoirement suspendue (en l'occurrence grâce à une neutralisation de type sceptique) afin que les contraintes de sa mise en discours puissent être objectivées et analysées. Il devient alors possible d'entrer dans le détail des modes de constitution discursive d'une pensée et ainsi d'augmenter son intelligibilité et parfois même de proposer des hypothèses interprétatives ou d'intervenir dans les débats d'histoire de la philosophie. L'objectivation des opérations qui permettent d'expliciter le rapport existant entre corpus, doctrine et œuvre philosophique, concernant ce qu'est une œuvre, voire un chef d'œuvre philosophique, ou concernant l'interprétation ne sont pas forcloses par l'analyse du discours philosophique : elles sont simplement reposées à nouveaux frais une fois conduite la phase d'objectivation. Cette analyse discursive une fois effectuée, rien en droit ne s'oppose à ce que la philosophie reprenne ses droits par des prises de position assumées qui seront armées par les résultats de l'analyse ${ }^{17}$. Ainsi une étude des dialogues de Platon ou des formes d'énonciation des sceptiques grecs pourra être mise au service d'une critique des «Ethiques du discours » comme celle de K.O. Apel (Cossutta 2003a). Il y a donc une boucle assurant un passage réglé de l'analyse du discours philosophique vers la philosophie, et vice versa.

\section{L'analyse du discours philosophique modifie notre regard sur la philosophie}

29 Appréhender la philosophie en analysant systématiquement ses dimensions discursives a pour effet de modifier le regard que nous portons sur elle et conduit à enrichir la description de ses caractéristiques. Et cela dans trois domaines, qui ne seront qu'esquissés ${ }^{18}$.

\subsection{Contraintes générales de mise en discours de l'activité philosophique}

Nombre de travaux, individuels ou réalisés collectivement dans le cadre du GradPhi, ont porté sur les contraintes auxquelles est soumis le discours philosophique, en relation avec des pratiques et des institutions historiquement situées.

On peut évoquer les recherches sur ce qu'on peut appeler "l'enceinte philosophique », l'espace institutionnel que présuppose la production philosophique et dont elle doit gérer les frontières. Cela concerne en particulier les rôles qu'assument les acteurs 
(Maingueneau 2019), les communautés philosophiques et les conflits à l'intérieur de ces communautés (Angermuller 2013 a, b, Fotuhi 2014). On évoquera également les travaux sur les institutions qui permettent et façonnent l'exercice de la pensée philosophique : traditions d'école orales et écrites, cursus d'enseignement de la philosophie, articulation des formes textuelles à des exercices et des pratiques mentales (Cossutta 2019a). Ce type d'approche donne tout leur poids aux genres "seconds", ceux qui ne visent pas à élaborer des doctrines. Dans le même ordre d'idées, une attention particulière a été accordée aux "formules philosophiques" (Maingueneau 2012, Cossutta \& Cicurel (éds.) 2014) qui sont étroitement liées à des pratiques d'apprentissage et d'interprétation et qui jouent un rôle important dans la circulation des énoncés philosophiques sur un vaste interdiscours.

Une telle démarche ne peut que subvertir la distinction traditionnelle entre la « vie » et l'« œuvre ", puisque l'institution philosophique fait éclater les termes mêmes de cette relation (Cossutta, Delormas \& Maingueneau (éds) 2012). Le philosophe structure sa «vie» à travers son «œuvre » et son «œuvre" à travers sa «vie» en fonction des normes qui prévalent dans l'institution philosophique à un moment donné.

\subsection{Mise en forme des modus operandi de l'énonciation philosophique}

D'autres travaux ont privilégié les contraintes sur la mise en forme de la pensée. Cela passe par l'étude de la mise en texte, en particulier à travers l'analyse de l'argumentation (Cossutta [éd.] 1996). Cela passe aussi par son articulation sur l'institution. Le concept en philosophie a ainsi pu être appréhendé à la fois comme point de condensation sémantique dans un texte et comme unité détachable, intégrée à des ouvrages d'ordre lexicographique qui contribuent à unifier le champ philosophique (Cossutta (éd.) 2019b). L'articulation entre texte et institution est plus immédiate quand on prend en compte les modes d'exposition et les cadres génériques; des études ont été consacrées à divers genres dialogiques : polémique (Ali Bouacha 2000), controverse (Fotuhi 2014), dialogue (Cossutta 2001, Cossutta (éd.) 2005), mais aussi à l'essai (Maingueneau 1998), à la méditation (Cossutta 1996). Le genre lui-même participe d'une scène d'énonciation (Maingueneau 2004) dont témoignent les multiples modalités de prise en compte des lecteurs.

\subsection{La spécificité du discours philosophique}

La philosophie se déploie en des unités de sens organisées et liées entre elles par lesquelles une doctrine, une pensée s'inscrivent dans des séries de textes (opus) qui se rassemblent dans une œuvre attachée à un nom d'auteur. On peut parler d'œuvre dans la mesure où la singularité d'une écriture, d'une façon d'agencer le propos est indissociable d'une singularité de pensée; avec ce paradoxe que le fait d'associer une philosophie à une identité auctoriale contingente n'ôte rien à l'universalité virtuelle de sa portée. Un certain nombre de travaux ont porté sur des œuvres philosophiques appréhendées sous l'angle de leur mode de constitution discursive : celles de Platon, de Descartes (Cossutta (éd.) 1996), Bergson (Cossutta (éd.) 1998), Leibniz et Levinas, Derrida (Maingueneau et Vallespir (éds) 2015). Cet effort pour analyser intégralement et en détail une œuvre dans le cadre d'une lecture plurielle permet non seulement 
d'éclairer la singularité d'un style philosophique, mais encore de comprendre le rapport entre une doctrine, le corpus où elle se déploie dans les divers moments de son actualisation, et l'œuvre ou les œuvres singulières par lesquelles elle acquiert un statut qui dépasse sa simple visée de vérité, au point de lui conférer la valeur d'un chef d'œuvre.

D'un point de vue d'analyse du discours, introduire la notion d'œuvre permet de rendre compte de certaines propriétés que la philosophie partage avec la littérature, mais ne suffit pas à rendre compte de sa spécificité (Maingueneau 2004, Cossutta 2003b). À cette fin, ont été élaborées les catégories de "discours constituant " et de "constituance " (Maingueneau et Cossutta 1995, Maingueneau 1999). Il fallait éviter, au moins à titre de précaution épistémologique, de conférer un privilège exorbitant à la philosophie, tout en expliquant ce qui dans son fonctionnement même a rendu possible la position de surplomb qu'elle s'est arrogée à l'égard d'autres discours. Et ceci vaut aussi pour des conceptions post-modernes, où, en apparence, elle a affiché des prétentions beaucoup plus modestes.

Ainsi, à nos yeux, le mérite principal de cette entreprise, est de contribuer à modifier le regard que nous portons sur la philosophie. Il ne s'agit plus seulement de doctrines, de systèmes de pensée déposés dans de grandes œuvres perpétuellement réinterprétées et dont il faudrait retracer le mode d'inscription dans une historicité elle-même philosophique. L'attention se déporte vers l'analyse des systèmes d'actes associant l'action de penser - avec les pratiques qui la rendent possible - et l'action d'écrire et de lire - en fonction des supports qui les rendent accessibles : oraux, écrits, imprimés, numériques.

\section{Les articles : entre analyses et bilan critique}

Le numéro que nous proposons rassemble sept contributions, qui se divisent en deux ensembles. Les quatre premières entendent illustrer la fécondité des méthodes d'analyse du discours philosophique sur des objets différents : paratexte, transmission, auctorialité, médiatisation ; les trois suivants en proposent une approche critique.

\subsection{La philosophie, son péritexte, ses contextes de production et de transmission}

Les contributions de F. Cossutta, D. Maingueneau, M. Temmar et J. Angermuller, soulignent l'intérêt d'une approche discursive sur plusieurs domaines qui ont en commun d'articuler les textes sur la dimension institutionnelle de la philosophie

La contribution de F. Cossutta aborde un objet qu'on pourrait de prime abord juger secondaire, en l'occurrence les préfaces et texte liminaires, qui relèvent selon lui d'un "agir préfacier " déterminant pour l'auto-constitution de la philosophie, mais aussi plus largement des discours théoriques. Les préfaces apparaissent en effet comme un espace de négociation qui permet de surmonter le risque de tensions entre les contraintes qu'imposent les visées de vérité et celles qu'imposent les visées communicationnelles. Elles négocient le mode d'inscription simultané de l'auteur et du lecteur ainsi que les conditions de la lecture, et configurent l'organisation du texte, de manière à donner à une doctrine la forme d'une œuvre. 
Pour D. Maingueneau, le discours philosophique ne se réduit pas à un patrimoine de textes: il faut élargir la perspective, prendre en compte l'espace où différentes catégories d'acteurs accomplissent des tâches spécifiques. Il distingue trois grands types de rôles: les "penseurs ", dont se soutient l'ensemble du discours, les "gestionnaires» qui stabilisent l'institution philosophique en balisant de multiples manières son archive, et les "vulgarisateurs" et les "essayistes » qui assurent la communication avec des publics extérieurs. Les "essayistes " sont placés dans une position ambiguë : ils produisent des textes qui se réclament de la philosophie mais privilégient les catégories et les préoccupations des non-experts. Ces diverses distinctions sont illustrées par des exemples empruntés à la philosophie contemporaine, en particulier à Michel Serres et Michel Onfray.

$41 \mathrm{M}$. Temmar s'intéresse à un autre type de médiation : celle qui résulte de l'interaction entre des philosophes et les journalistes. Elle aborde en effet un corpus original, délaissé aussi bien par les philosophes que par les analystes du discours: la parole philosophique dans les médias, plus particulièrement à travers les entretiens avec des philosophes que publie la presse écrite. Elle s'attache à montrer que ce type d'entretien présente des spécificités qui renvoient au statut privilégié qu'occupe le philosophe, distinct d'autres experts, comme les sociologues ou les politistes. L'étude s'appuie sur un corpus: les interventions de philosophes sollicités pour réagir à une question d'actualité, en l'occurrence les attentats terroristes. Le philosophe, traditionnellement voué à prendre de la distance, y est confronté à une demande de sens urgente de la part de la société.

J. Angermuller, comme D. Maingueneau, s'intéresse à la communauté des philosophes, mais du point de vue des acteurs eux-mêmes. La philosophie est perçue comme une pratique discursive où les philosophes se positionnent et se catégorisent les uns les autres en tant que membres d'une même communauté. Pour illustrer son propos il revient sur la controverse célèbre entre Derrida et Foucault. À travers cet échange qui semble purement théorique sur la manière dont il faut comprendre la position de Descartes par rapport à la folie, chacun des deux effectue une mise en scène énonciative qui définit sa relation à l'institution philosophique. L'analyse révèle ainsi le caractère indissociablement social et intellectuel de leur controverse, la construction divergente de leurs positions de sujet dans la communauté intellectuelle parisienne des années 1960 et 1970.

Ces quatre contributions ont en commun de ne pas se focaliser sur ce qui constitue traditionnellement le fond de l'activité philosophique: le commentaire des "grands textes ». Chacune à sa façon, elles décalent le regard: des textes au péritexte (Cossutta), des penseurs aux gestionnaires et aux médiateurs (Maingueneau), des textes proprement philosophiques aux textes médiatiques (Temmar), des purs penseurs aux membres d'une communauté académique (Angermuller). Par là il ne s'agit pas de se placer à « l'extérieur » de la philosophie, de substituer à l'étude des textes canoniques l'étude de textes périphériques, de faible valeur doctrinale, mais de passer de la seule étude du Thésaurus de grands textes couplée avec des travaux d'ordre historique, à une prise en compte du discours philosophique, envisagé dans la complexité de son fonctionnement, en contestant toute opposition simple entre un intérieur et un extérieur du texte philosophique, un intérieur et un extérieur de la philosophie ellemême. Les préfaces ne sont pas des suppléments contingents, mais une zone aussi incertaine qu'essentielle: une préface est signée de "l'auteur», catégorie qui 
précisément n'existe que sur une frontière, celle entre le texte et le monde où il est appelé à prendre place. Les philosophes ne sont pas de simples énonciateurs, mais assument des rôles qui délimitent et structurent l'espace de la philosophie. La médiatisation et l'image du philosophe sont partie intégrante de la philosophie, qui ne cesse de légitimer son existence et ses acteurs auprès de la société. La communauté des philosophes n'est pas un lieu où émergeraient des doctrines qui en sont indépendantes, mais la condition même de ces doctrines, qui ne cessent de la réfléchir de manière oblique.

\subsection{Mises en perspective critiques de l'analyse du discours philosophique}

La seconde série de contributions met en perspective l'analyse du discours philosophique elle-même, à travers des points de vue différents. C'est sa démarche même qui est réfléchie, en relation avec d'autres approches de la textualité philosophique: la philosophe de la déconstruction (C. Thevenet), la stylistique (A. Lhomme), l'herméneutique (M. Vallespir). Ce qui permet de mettre en évidence certains retours de présupposés philosophiques dans l'analyse du discours philosophique. Si nous avons ainsi fait une place à ces articles, c'est qu'il nous a semblé important de ne pas replier sur elle-même, comme une simple discipline empirique, une analyse du discours philosophique dont, en réalité, le statut ne peut qu'être problématique.

C. Thevenet confronte l'analyse du discours à la déconstruction derridienne. Elle part $\mathrm{du}$ constat que l'analyse du discours vise à forger des catégories permettant de lire et de décrire les opérations discursives dans les œuvres de philosophie, mais que certains textes semblent résister à l'analyse. C'est le cas en particulier de ces livres de Derrida, Glas (1974) ou Feu la cendre (1986), disposés sur deux colonnes. Plutôt que de conclure à l'impuissance de l'analyse du discours philosophique à les analyser, l'article se propose de relativiser la supposée incompatibilité de l'analyse du discours philosophique et du corpus derridien. Après avoir souligné leurs affinités, elle s'efforce de redéfinir la notion de «ton" à partir de deux textes de Derrida - D'un ton apocalyptique adopté naguère en philosophie et de Feu la cendre - en vue de son emploi dans le champ de l'analyse du discours philosophique.

A. Lhomme adopte, lui, un point de vue délibérément critique à l'égard de l'analyse du discours, en ce sens qu'il lui assigne des limites. Tout en prenant acte de ce qu'apporte l'application aux textes des catégorise de l'analyse du discours, il considère que certaines dimensions du texte échappent à une approche proprement discursive, que le texte résiste à un alignement sur le discours. Il défend les vertus d'une analyse proprement textuelle, mieux à même selon lui de prendre en charge les fonctions métaargumentatives et conceptuellement thématisantes que remplissent les textes philosophiques. Une démarche qui implique toutefois un renouvellement de la stylistique philosophique, dont il esquisse le programme.

La perspective de $\mathrm{M}$. Vallespir est très différente, puisqu'elle ne conteste pas de l'extérieur l'analyse du discours philosophique mais réfléchit sur son statut : est-ce une discipline à part entière? S'agit-il d'une branche de l'analyse du discours ou d'une modification des présupposés de cette discipline qu'est l'analyse du discours? Elle interroge ainsi la relation du corpus philosophique à l'analyse du discours, à ses 
catégories et ses méthodes. En s'appuyant sur le fonctionnement du Groupe d'Analyse du Discours Philosophique, elle propose une "cartographie" de cette dernière, envisagée comme activité autant théorique que pratique qui opère une redéfinition tant de l'analyse du discours que de la philosophie.

Ces trois contributions, on le voit, ont des présupposés et des visées très différents. Tout en intégrant certains apports de l'analyse du discours, A. Lhomme lui impose une limite de droit, pour ménager l'espace d'une autre approche, qui se propose de prolonger, en la renouvelant, une certaine tradition. Il se situe donc à l'extérieur, et loin de contester l'opposition entre texte et contexte, la radicalise. En revanche, C. Thevenet et $\mathrm{M}$. Vallespir libèrent des espaces à l'intérieur de l'analyse du discours philosophique, ils la font jouer sur elle-même en la confrontant à des démarches dont elles montrent qu'elles ne peuvent être renvoyées purement et simplement hors de ses frontières. C. Thevenet va ainsi vers un décloisonnement entre la méthode d'investigation (analyse du discours) et l'objet étudié (l'œuvre de Derrida en l'occurrence).

\section{BIBLIOGRAPHIE}

Adam. Jean-Michel. 2008. «La phrase dans le discours. Autour de quelques réénonciations du cogito cartésien », O. Brand et al. (éds), Discours, diachronie, stylistique du français. Etudes en hommage à Bernard Combette (Bern : Peter Lang), 3-16

Adam, Jean-Michel. 2011. La linguistique textuelle. Introduction à l'analyse textuelle des discours (Paris : A. Colin)

Adam, Jean-Michel. 2015. «L'analyse textuelle des discours. Entre linguistique textuelle et analyse du discours ", L'analyse de discours. Sa place dans les sciences du langage et de la communication. Hommage à Patrick Charaudeau (Rennes : Presses Universitaires de Rennes),19-26

Ali Bouacha, Magid. 1995. «De l'ego à la classe de locuteurs : lecture linguistique des Méditations ", Langages 119, 79-94

Ali Bouacha, Magid. 2000. « l'AUTRE du sujet dans 'La recherche de la vérité par la lumière naturelle' de Descartes ", Ali Bouacha, Magid \& Frédéric Cossutta (éds) La polémique en philosophie. La polémicité et ses mises en discours (Dijon : Editions Universitaires de Dijon), 19-37

Ali Bouacha, Magid, \& Frédéric Cossutta. (éds). 2000. La polémique en philosophie (Dijon : Editions Universitaires de Dijon)

Ali Bouacha, Magid. 2017. « Les écrits de Bachelard : entre fictivité et fiction », José Agular, Lucile Cadet, Catherine Muller \& Véronique Rivière (éds). L'enseignant et le chercheur au cœur des discours, des textes et des actions. Mélange offerts à Francine Cicurel (Paris : Riveneuve Editions), 21-40

Amossy, Ruth. 2000. L'argumentation dans le discours : discours politique, littérature d'idées, fiction (Paris : Nathan). Rééditions : Paris, A. Colin, coll. « Cursus », 2005 ; coll. « I.COM », 2013.

Angermuller, Johannes. 2013a. Analyse du discours poststructuraliste. Les voix du sujet dans le langage chez Lacan, Althusser, Foucault, Derrida et Sollers (Limoges : Lambert Lucas) 
Angermuller, Johannes. 2013b. Le Champ de la Théorie. Essor et déclin du structuralisme en France (Paris : Hermann)

Bianco, Giuseppe. 2015. Après Bergson : portrait de groupe avec philosophe (Paris : PUF)

Bordron, Jean-François. 1987. Descartes. Recherches sur les contraintes sémiotiques de la pensée discursive (Paris : PUF)

Bordron, Jean-François. 2011. L'iconicité et ses images (Paris : PUF)

Bordron, Jean-François. 2016. Le discours spéculatif. Approche sémiotique (Limoges : Lambert-Lucas)

Botet, Serge. 2006. Le Zarathoustra de Nietzsche : une refonte du discours philosophique? (Paris :

Klincksieck)

Cahné, Pierre-Alain. 1980. Un autre Descartes. Le philosophe et son langage (Paris : Vrin)

Cicurel, Francine. 2014. « Mot d'ordre ou mot de passe : les formes textuelles de la transmissibilité en philosophie », Frédéric Cossutta \& Francine Cicurel (éds). Les formules philosophiques. Détachement, circulation, recontextualisation (Limoges : Lambert-Lucas), 111-130

Clément, Bruno. 2000. L'invention du commentaire : Augustin, Jacques Derrida (Paris : PUF)

Clément, Bruno. 2005. Le récit de la méthode (Paris : Seuil)

Clément, Bruno. 2013. La Voix verticale - Essai sur la prosopopée (Paris : Belin)

Collins, Randall. 1998. The Sociology of Philosophies. A Global Theory of Intellectual Change (Cambridge, Massachusetts, and London : The Belknap Press of Harvard University Press)

Cossutta, Frédéric. 1989. Éléments pour la lecture des textes philosophiques (Paris : Bordas)

Cossutta, Frédéric. 1995. « Pour une analyse du discours philosophique », Langages 119, 12-39

Cossutta, Frédéric. 1996. «À quelles conditions une théorie de l'argumentation philosophique est-elle possible? », Cossutta, Frédéric (éd.). Descartes et l'argumentation philosophique (Paris : PUF), $1-42$

Cossutta, Frédéric. 1998. «L'analyse du discours philosophique », Mattéi, J.-F. (éd.). Encyclopédie philosophique Universelle, t. IV, Le Discours philosophique (Paris : PUF), 1792-1810

Cossutta, Frédéric. 2001. «Les formes en philosophie : le dialogue. Etude de son emploi par Descartes et Leibniz », Styles et genres en philosophie, Cahiers philosophiques 89, 66-89

Cossutta, Frédéric. 2003a. « Pour une critique sceptique de la pragmatique transcendantale de K.O. Apel », Methodos 3, 251-288

Cossutta, Frédéric. 2003b. « Discours littéraire, discours philosophique, deux formes d'autoconstitution? » Ruth Amossy \& Dominique Maingueneau (éds), L'analyse du discours dans les études littéraires (Toulouse : Presses Universitaires du Mirail), 417-428

Cossutta, Frédéric. 2019a « Le style diatribique dans les Diatribai d'Epictète/Arrien », Favreau Anne-Marie \& Jean-Pierre de Giorgio, Les diatribes dans l'antiquité: des dialogues comme les autres? (Limoges : Lambert-Lucas), 65-86

Cossutta, Frédéric (éd). 1998. Lire Bergson, «le possible et le réel » (Paris : PUF)

Cossutta, Frédéric (éd.). 2005. Le Dialogue : introduction à un genre philosophique (Villeneuve d'Ascq : Presses Universitaires du Septentrion)

Cossutta, Frédéric, Pascale Delormas \& Dominique Maingueneau (éds). 2012. La Vie à l'œuvre, le biographique dans le discours philosophique (Limoges : Lambert-Lucas) 
Cossutta, Frédéric \& Francine Cicurel (éds). 2014. Les Formules philosophiques (Limoges : LambertLucas)

Cossutta, Frédéric. (éd.). 2019b, à paraître. La fabrique discursive des concepts philosophiques (Limoges : Lambert-Lucas)

Curatolo, Bruno \& Jacques Poirier (éds). 2007. Le style des philosophes (Dijon : Editions Universitaires de Dijon et Presses Universitaires de Franche-Comté)

Delormas, Pascale. 2013. De l'autobiographie à la mise en scène de soi. Le cas Rousseau (Limoges : Lambert-Lucas)

Derrida, Jacques. 1998. « A quoi pensent les philosophes », Autrement 102, 30-37

Ducard, Dominique. 2019. «Terme, notion, concept. L'entreprise du 'Vocabulaire de Lalande' ", Cossutta Frédéric (éd.), 2019. La fabrique discursive des concepts philosophiques (Limoges : LambertLucas) (à paraître)

Fabiani, Jean-Louis. 2010. Qu'est-ce qu'un philosophe français? La vie sociale des concepts (1880-1980) (Paris : Editions de l'EHESS)

Fotuhi, Khodayar. 2014. « Entre Foucault et Derrida : le sort d'un énoncé cartésien », Frédéric Cossutta et Francine Cicurel (éds.), Les Formules philosophiques (Limoges, Lambert-Lucas 140-159 Gadamer, Hans-Georg. 1976. Vérité et Méthode. Les grandes lignes d'une herméneutique philosophique, (Paris : Seuil)

Giolito, Christophe. 1999. Histoires de la philosophie avec Martial Gueroult (Paris : L’Harmattan) Granger, Gilles-Gaston. 1988. Pour la connaissance philosophique (Paris : Odile Jacob)

Grize, Jean-Blaise (éd.). 1984. Sémiologie du raisonnement (Bern : Peter Lang)

Gueroult, Martial. 1979. Dianoématique 1, Philosophie de l'histoire de la philosophie (Paris : AubierMontaigne)

Goldschmidt, Victor. 1971. Les dialogues de Platon (Paris : PUF)

Hadot, Pierre. 1987. Exercices spirituels et philosophie antique (Paris : Etudes Augustiniennes)

Lhomme, Alain. 1998. « Le style des philosophes », Jean-François Mattéi (éd.). Le Discours

philosophique. Encyclopédie philosophique universelle (Paris : PUF), 1564-1583

Lhomme, Alain. 2000. « Pensées fictives et fictions de l'imagination. Sur l'écriture de la fiction dans les Méditations métaphysiques de Descartes ", Littérature et philosophie, Europe 849/850, 184-200

Lhomme, Alain. 2005. « Entre concept et métaphore : existe-t-il une écriture spécifiquement philosophique? ", L'Écriture des philosophes, Rue Descartes 50, 59-73

Loraux, Patrice. 1993. Le tempo de la pensée (Paris : Seuil)

Macherey, Pierre. 1990. A quoi pense la littérature? (Paris : PUF)

Maingueneau, Dominique. 1995. «L'Analyse des discours constituants » (en collaboration avec Frédéric Cossutta), Langages 117, 112-125

Maingueneau, Dominique. 1995. « Le discours philosophique comme institution discursive », Langages 119, 40-62

Maingueneau, Dominique. 1998. « "'Le possible et le réel' de Bergson, un texte mineur ?», Cossutta, Frédéric (éd.) (Paris : PUF), 29-48 
Maingueneau, Dominique. 1999. « Analyzing self-constituting discourses », Discourse studies 1 (2), 175-199

Maingueneau, Dominique. 2004. Le discours littéraire. Paratopie et scène d'énonciation. (Paris : A. Colin)

Maingueneau, Dominique. 2012. Les phrases sans texte (Paris : A. Colin)

Maingueneau, Dominique. 2015. La philosophie comme institution discursive (Limoges : LambertLucas)

Maingueneau, Dominique. 2019. «Cartographie philosophique et extraction de concepts ", Frédéric Cossutta (éd.). La fabrique discursive des concepts philosophiques (Limoges : Lambert-Lucas)

Maingueneau, Dominique \& Mathilde Vallespir (éds). 2015. Lire Derrida? Autour d’Éperons. Les styles de Nietzsche (Limoges : Lambert-Lucas)

Paveau, Marie-Anne. 2013. Langage et morale. Une éthique des vertus discursives (Limoges : LambertLucas)

Perelman, Chaïm \& Lucie Olbrechts-Tyteca 2008 [1970]. Traité de l'argumentation. La nouvelle rhétorique (Bruxelles : Editions de l'Université de Bruxelles)

Pinto, Louis. 2007. La Vocation et le métier de philosophe. Pour une sociologie de la philosophie dans la France contemporaine (Paris : Seuil)

Pinto, Louis. 2014. Sociologie et philosophie : libres échanges. Bourdieu, Durkheim, Foucault, Sartre... (Paris : Ithaque)

Plantin, Christian. 2016. Dictionnaire de l'argumentation. Une introduction aux études d'argumentation (Lyon : ENS éditions)

Robinet, André. 1986. Architectonique disjonctive, automates systémiques et idéalité transcendantale dans l'œuvre de G. W. Leibniz, (Paris : Vrin)

Ricœur, Paul. 1986. Du texte à l'action. Essais d'herméneutique II (Paris : Seuil)

Ryle, Gilbert. 1971. «Systematically Misleading Expressions », Collected Papers vol 2, Collected Essays (London : Hutchinson), 39-62

Ryle, Gilbert. 1978 [1949). La notion de l'esprit (Paris : Payot)

Sabot, Philippe. 2001. Pratiques d'écriture, pratiques de pensée : Figures du sujet chez Breton, Eluard, Bataille et Leiris (Lille : Presses Universitaires du Septentrion)

Schneider, U. J. 1990. Die Vergangenheit des Geistes. Eine Archäologie der Philosophiegeschichte (Frankfurt a. M. : Suhrkamp)

Schneider, Ulrich Johannes. 1996 « 'Ein Dilemma tiefster Schwierigkeiten'. Zur Theorie der Philosophiegeschichte im 20. Jahrhundert », Klaus-Dieter Eichler und Volker Caysa (eds), Philosophiegeschichte und Hermeneutik (Leipzig : Leipziger Universitätsverlag), 46-69

Serres, Michel. 1968. Le système de Leibniz et ses modèles mathématiques (Paris : PUF)

Soulez, Antonia. 2003. Comment écrivent les philosophes? (De Kant à Wittgenstein) ou le style Wittgenstein (Paris : Editions Kimé)

Souriau, Etienne. 1939. L'Instauration philosophique (Paris : Alcan)

Temmar, Malika. 2013. Le recours à la fiction dans le discours philosophique (Limoges : Lambert-Lucas) 
Vuillemin, Jules. 1984. Nécessité ou contingence. L'aporie de Diodore et les systèmes philosophiques

(Paris : Les éditions de Minuit)

\section{NOTES}

1. Voir, déjà, Cossutta 1989 : «Introduction ».

2. Faute de pouvoir citer, dans cet espace restreint ces travaux sur le discours philosophique, nous invitons le lecteur à se reporter, sur le site du Groupe aux pages personnelles des membres, auxquelles sont associées des bibliographies complètes (https://gradphi.hypotheses.org) .

3. Le Groupe de rechercher sur l'analyse du discours philosophique a été créé sur l'idée d'une pluralité des modes d'investigation du discours philosophique. Par exemple, J.-F. Bordron se réclame de la sémiotique (Bordron 2016), A. Lhomme, (Lhomme 1998, 2000, 2005), comme en témoigne sa contribution ici même, fait appel à une stylistique et $\mathrm{B}$. Clément à une théorie des figures (Clément 2013).

4. Illustré par différents numéros de la revue Langages, en particulier les $n^{\circ} 13,55,81$, et pour ce qui nous intéresse ici, par le $n^{\circ} 117$ (1995), Les analyses du discours en France, dirigé par D. Maingueneau.

5. Voir Maingueneau 2015, Angermulller 2013, Delormas 2013, Temmar 2013.

6. Nos travaux n'ont en effet rencontré que très peu d'intérêt dans la communauté des philosophes et n'ont pas donné lieu à de substantielles réappropriations ou prolongements, si l'on excepte des collaborations ponctuelles fructueuses. Ni le CNRS ni l'Université n'ont accordé la moindre légitimité institutionnelle à ce type de recherche.

7. Sur l'œuvre de Gueroult, voir Giolito 1999.

8. La philosophie française a-t-elle l'esprit de système?, Les Temps Modernes, 2015, nº 682.

9. Cette question a donné lieu à une littérature importante dont il n'est pas question de rendre compte ici.

10. L'explicitation des conditions de possibilité d'une analyse du discours philosophique suppose de surmonter les limites, pour ne pas dire les impasses que représentent l'herméneutique d'un côté, la déconstruction de l'autre si on se donne un autre objectif. Cf. Cossutta (1998: 1795) : « Si, Ricœur, alors Derrida, si, Derrida, alors Ricœur. »

11. Sans oublier le livre fondateur aux USA de R. Collins qui n'a jamais été traduit (Collins 1998), en France on citera en particulier les travaux de sociologie de la philosophie menés par J.-L. Fabiani (2010), L. Pinto (2007, 2014), et G. Bianco (2015).

12. Il est significatif que J.-L. Fabiani (2010), après avoir commencé par poser la nécessité de subvertir la distinction entre texte et contexte y revienne subrepticement: dans un premier temps il analyse les institutions de la philosophie française pour ensuite s'intéresser au contenu des doctrines, qu'il s'efforce de classer. Notre démarche consiste au contraire à essayer de nous installer dans la zone intermédiaire.

13. Voir, à titre d'exception, sur le cogito, Adam $2015: 19-22$.

14. Pour une articulation un peu différente entre « institution » et « instauration » discursive de la philosophie, voir aussi Cossutta (1995: 69), (2001: 71).

15. Les travaux d'U. J. Schneider qui participent de cette diversité $(1990,1996)$ n'ont pas été traduits et son méconnus en France.

16. Voir l'article manifeste du numéro de Langages 119: "Pour une analyse du discours philosophique » dont la première section est intitulée : "À quelles conditions une analyse du discours philosophique est-elle possible? ? (Cossutta 1995 : 12-23).

17. Nous ne pouvons en cela qu'être encouragés par les extrapolations philosophiques auxquelles l'analyse du discours peut donner lieu. Elle ne s'est pas interdit une dimension critique ou normative d'un point de vue politique ou idéologique lors de son développement en France. Ce 
qui a entraîné une réflexion épistémologique sur ses propres fondements et son rapport aux questions normatives et axiologiques comme en témoigne le numéro d'Argumentation et analyse du discours dirigé par R. Koren consacré à l'engagement du chercheur $\left(n^{\circ} 11,2013\right)$. Certains analystes du discours ont fait preuve d'ailleurs d'une belle audace spéculative, comme M.-A. Paveau, dont le livre Langage et morale. Une éthique des vertus discursives est "consacré à l'élaboration des conditions de l'intégration de la dimension morale à l'analyse linguistique des discours, en particulier à travers la notion de vertu discursive » (Paveau $2013: 4^{\text {ème }}$ de couv.).

18. La bibliographie raisonnée, située à la fin du numéro, donne une idée plus précise des travaux qui ont été conduits dans ces divers domaines.

\section{AUTEURS}

\section{FRÉDÉRIC COSSUTTA}

CEDITEC, Paris-Est Val de Marne

DOMINIQUE MAINGUENEAU

Sorbonne Université 